\title{
EXTENDED UV EMISSION IN NGC6251
}

\author{
PHILIPPE CRANE AND JOEL VERNET \\ European Southern Observatory \\ Garching, Germany
}

\section{Introduction}

NGC 6251 is an optically normal elliptical galaxy that hosts one of the most spectacular radio jets(Perley, Bridle, and Willis, 1984). This jet extends about $1 \mathrm{Mpc}$ and its axis is roughly perpendicular to the central dust ring. NGC6251 was originally observed with HST in a survey of elliptical galaxies(Crane et $a l ., 1993)$ and revealed an anamoly which was pursued in the investigation reported here.

Hubble Space Telescope Images of the nucleus of the galaxy NGC6251 have been obtained with FOC F/96 camera at 3 different blue and UV filters. Images with the PC2 camera at 2 wavelengths have been extracted from the HST archives. The PC images reveal a $500 \mathrm{pc}$ diameter warped dust ring surrounding the nucleus of NGC 6251. However, the FOC images reveal a bright extension to the nucleus apparently filling the interior of the dust ring, and which is seen best at wavelengths shortward of $\lambda \leq \approx 4000$ A. (See figure 1).

Our previous report(Crane and Vernet, 1997) on this discovery did not include the image at $2200 \AA$ which is seen in the upper left hand corner of Fig. 1. This new image clearly supports our previous assertion that this emission is most likely scattered radiation which originates at or near the true nucleus.

\section{Discussion}

We have no definitive explanation for these observations. Nevertheless, several lines of reasoning have led us to favor the interpretation of the radiation as being scattered even though our polarization images do not show substantial polarization. ( See Crane and Vernet(1997) for details.) 

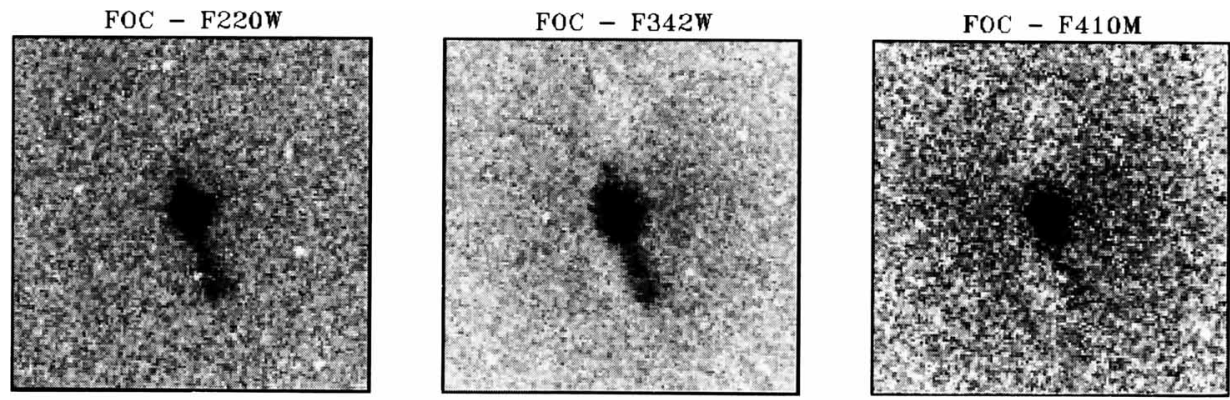

WFPC2 - F555W

WFPC2 - F814W
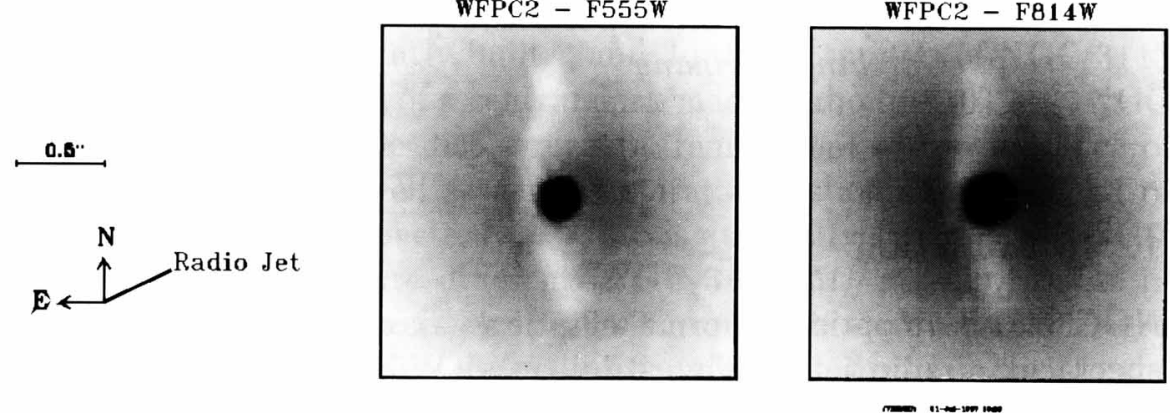

Figure 1. HST Images of the central few arcseconds of NGC 6251. The HST filters used for each image are indicated. The wavelengths progresses from $2200 \AA$ in the upper left to $8000 \AA$ in the lower right. The orientation and scale are indicated in the lower left panel. The arrow indicates the direction of the radio jet.

The canonical models of the close regions of galactic nuclei usually propose an optically thick torus in the inner parsec or so. This torus presumably prevents our view of the interior regions except for a restricted range of viewing angles. However, recently Blackman and Yi(1996) have proposed that this torus may be supressed or even absent in sources with very powerful radio sources. We suggest that this may be the case for NGC 6251 .

\section{References}

Blackman, E. and Yi,I.,(1996)Ap.J.461 L21.

Crane,P.,et al.,(1993)AJ. 106, 1371.

Crane,P., and Vernet, J.,(1997)Ap.J. 496,L91.

Perley, R.A., Bridle, A.H., and Willis, A.G.,(1984)Ap.JS., 54,291. 